\title{
1 A molecular barcode and online tool to identify and map imported infection
}

\section{2 with Plasmodium vivax}

3 Hidayat Trimarsanto ${ }^{1,2}$, Roberto Amato ${ }^{3,4}$, Richard D Pearson ${ }^{3,4}$, Edwin Sutanto ${ }^{1}$, Rintis Noviyanti ${ }^{1}$, Leily

4 Trianty $^{1}$, Jutta Marfurt ${ }^{5}$, Zuleima Pava ${ }^{5}$, Diego F Echeverry ${ }^{6,7}$, Tatiana M Lopera-Mesa ${ }^{8}$, Lidia Madeline

5 Montenegro $^{8}$, Alberto Tobón-Castaño ${ }^{8}$, Matthew J Grigg ${ }^{5,9}$, Bridget Barber $^{5,9}$, Timothy William ${ }^{9,10}$,

6 Nicholas M Anstey ${ }^{5}$, Sisay Getachew ${ }^{11,12}$, Beyene Petros $^{11}$, Abraham Aseffa ${ }^{12}$, Ashenafi Assefa ${ }^{13}$, Awab

7 Ghulam Rahim ${ }^{14,15}$, Nguyen Hoang Chau ${ }^{16}$, Tran Tinh Hien ${ }^{16}$, Mohammad Shafiul Alam ${ }^{17}$, Wasif A Khan ${ }^{17}$,

8 Benedikt Ley ${ }^{5}$, Kamala Thriemer ${ }^{5}$, Sonam Wangchuck ${ }^{18}$, Yaghoob Hamedi ${ }^{19}$, Ishag Adam ${ }^{20}$, Yaobao

$9 \mathrm{Liu}^{21,22}$, Qi Gao ${ }^{22}$, Kanlaya Sriprawat ${ }^{23}$, Marcelo U Ferreira ${ }^{24}$, Alyssa Barry ${ }^{25,26,27,28}$, Ivo Mueller ${ }^{28,29}$, Eleanor

10 Drury $^{4}$, Sonia Goncalves ${ }^{4}$, Victoria Simpson ${ }^{3,4}$, Olivo Miotto ${ }^{3,4,13}$, Alistair Miles ${ }^{3,4}$, Nicholas J White ${ }^{13,30}$,

11 Francois Nosten ${ }^{13,23,30}$, Dominic P Kwiatkowski ${ }^{3,4}$, Ric N Price ${ }^{* 5,13,30}$, Sarah Auburn *5,13,30 Indonesia

3. Big Data Institute, Li Ka Shing Centre for Health Information and Discovery, Old Road Campus, Oxford, OX3 7LF, UK

4. Wellcome Sanger Institute, Hinxton, Cambridge CB10 1SA, UK

5. Global and Tropical Health Division, Menzies School of Health Research and Charles Darwin University, Darwin, Northern Territory 0811, Australia

6. Centro Internacional de Entrenamiento e Investigaciones Medicas, CIDEIM, Cali, Colombia

7. Universidad Icesi, Cali, Colombia

8. Grupo Malaria, Facultad de Medicina, Universidad de Antioquia, Medellin, Colombia 
9. Infectious Diseases Society Sabah-Menzies School of Health Research Clinical Research Unit, Kota Kinabalu, Sabah, Malaysia

10. Clinical Research Centre, Queen Elizabeth Hospital, Sabah, Malaysia Dist.5, Ho Chi Minh City, Vietnam Mohakhali, Dhaka, 1212, Bangladesh

18. Royal Center for Disease Control, Department of Public Health, Ministry of Health, Thimphu, Bhutan

19. Infectious and Tropical Diseases Research Center, Hormozgan University of Medical Sciences, Bandar Abbas, Hormozgan Province, Iran and Prevention, Jiangsu Provincial Key Laboratory on Parasite and Vector Control Technology,

48 25. School of Medicine, Deakin University, Geelong, VIC, Australia 
26. Burnet Institute, Melbourne, VIC, Australia

50

51

52

53

27. Population Health and Immunity Division, The Walter and Eliza Hall Institute of Medical Research, Parkville, VIC, Australia

28. Department of Medical Biology, The University of Melbourne, Parkville, VIC, Australia

29. Department of Parasites and Insect Vectors, Institut Pasteur, Paris, France

30. Centre for Tropical Medicine and Global Health, Nuffield Department of Medicine, University of Oxford, OX3 7L, UK

*Corresponding author: Dr Sarah Auburn, Menzies School of Health Research, PO Box 41096, Casuarina, Darwin, NT 0811, Australia; Tel: (+61) 889468503

\section{Abstract}

Imported cases present a considerable challenge to the elimination of malaria. Traditionally, patient travel history has been used to identify imported cases, but the long-latency liver stages confound this approach in Plasmodium vivax. Molecular tools to identify and map imported cases offer a more robust approach, that can be combined with drug resistance and other surveillance markers in highthroughput, population-based genotyping frameworks. Using a machine learning approach incorporating hierarchical FST (HFST) and decision tree (DT) analysis applied to $831 P$. vivax genomes from 20 countries, we identified a 28-Single Nucleotide Polymorphism (SNP) barcode with high capacity to predict the country of origin. The Matthews correlation coefficient (MCC), which provides a measure of the quality of the classifications, ranging from -1 (total disagreement) to 1 (perfect prediction), exceeded 0.9 in 15 countries in cross-validation evaluations. When combined with an existing 37-SNP $P$. vivax barcode, the 65-SNP panel exhibits MCC scores exceeding 0.9 in 17 countries with up to 30\% missing data. As a secondary objective, several genes were identified with moderate MCC scores 
73 (median MCC range from 0.54-0.68), amenable as markers for rapid testing using low-throughput

74 genotyping approaches. A likelihood-based classifier framework was established, that supports analysis

75 of missing data and polyclonal infections. To facilitate investigator-lead analyses, the likelihood

76 framework is provided as a web-based, open-access platform (vivaxGEN-geo) to support the analysis

77 and interpretation of data produced either at the 28-SNP core or full 65-SNP barcode. These tools can

78 be used by malaria control programs to identify the main reservoirs of infection so that resources can be

79 focused to where they are needed most.

\section{Keywords}

82 Plasmodium vivax, malaria, imported malaria, geographic origin, surveillance, genotyping, genomics,

83 molecular barcode

\section{Background}

86 The last three World Malaria Reports have revealed a disturbing rise in malaria cases, and, outside

87 Subsaharan Africa, an increasing proportion of malaria due to Plasmodium vivax, undermining the

88 painstaking efforts to reduce transmission over the past decade ${ }^{1}$. These trends highlight the urgent

89 need for new surveillance tools, with greater attention to the non-falciparum species. In today's global

90 climate, human populations are highly mobile, with imported cases of malaria confounding local control

91 efforts and enhancing the risks of drug resistance spread and outbreaks. There is thus a critical need to

92 develop tools that can help to determine where patients acquired their infection. 
93 The challenge of imported infections is particularly pertinent for $P$. vivax, in view of the parasite's ability

94 to form dormant liver stages (hypnozoites) that can reactivate weeks to months after the initial

95 infection, as well as highly persistent, low density blood-stage infections ${ }^{2,3}$. The re-emergence of $P$. vivax

96 in multiple regions where it was once almost eliminated serves as an important reminder of the need to

97 maintain diligent surveillance of this species ${ }^{4}$. In low endemic settings where the incidence of local

98 infections is declining, the relative proportion of imported cases generally rises, emphasizing the

99 importance for surveillance tools that can identify imported $P$. vivax cases. Traditionally, imported cases

100 have been identified and mapped using information on patient travel history, but the persistent blood

101 stage infections and long-latency liver stages constrain the accuracy of this approach in $P$. vivax

102 infections. Molecular tools to identify and map imported $P$. vivax cases offer an attractive complement

103 to traditional epidemiological tools.

104 Amplicon-based sequencing has become a favored approach for targeted genotyping of malaria

105 parasites. Using highly parallel sequencing platforms, such as the latest generation of Illumina

106 sequencers, amplicon-based sequencing can be applied at moderate to high-throughput, with high

107 accuracy and sensitivity. These platforms are flexible, allowing iterative enhancement of the Single

108 Nucleotide Polymorphism (SNP) barcodes, which can provide an affordable genotyping approach,

109 amenable to population-based molecular surveillance.

110 Previous studies have used mitochondrial and apicoplast markers to resolve imported from local P. vivax

111 isolates, but the resolution of these organelles is constrained ${ }^{5-7} \cdot \ln 2015$, a panel of 42 SNPs was

112 identified to facilitate parasite finger-printing and geographic assignment ${ }^{8}$. The proposed 42-SNP Broad

113 barcode was derived from genomic data available from 13 isolates from 7 countries. In the last 4 years,

114 the repository of genomic data on $P$. vivax has expanded greatly, allowing further refinement of a

115 parsimonious and widely applicable genotyping barcode. 
116 The primary objective of our study was to develop molecular tools for identifying and characterizing

117 imported P. vivax cases amenable to population-based surveillance frameworks, so that these data can

118 be used to inform strategic decisions on where and how to deploy malaria control interventions. We

119 tailored our molecular tools primarily to surveillance frameworks using Illumina or other high-

throughput genotyping platforms. As a secondary objective, we sought to identify single gene regions

121 permissible to lower throughput approaches for use in settings or situations where high-throughput or

122 centralized approaches are not feasible. In addition, we provide informatics tools to support users in

123 analyzing genotyping data produced at the barcode that can accommodate missing data and polyclonal

124 infections.

\section{Materials and Methods}

\section{Overview of the marker selection approach}

129 A flow diagram outlining the steps involved in the marker selection process is provided in Figure 1a. In

130 accordance with the multiplexing features of the Illumina platform, we sought to identify approximately

13150 new SNP-based markers to append to the recently published Broad barcode ${ }^{8}$, to provide a composite

132 panel with $\leq 100$ markers for country-level geographic assignment of $P$. vivax infections. The decision to

133 append markers to the Broad barcode rather than selecting a de novo panel of SNPs was pragmatic,

134 aimed at promoting consensus and continuity with existing molecular tools available to the vivax

135 community. A likelihood-based classifier approach was chosen for the respective evaluation of marker

136 sets and end-user data analysis tasks. This approach was chosen since it allows manual addition of

137 specific SNP sets, such as the Broad barcode. Two selector algorithms, hierarchical FST (HFST) and

138 decision-tree (DT), were implemented in the likelihood-based classifier framework to select SNPs with

139 high country-level prediction values. The primary SNP selection method was the HFST selector, which

140 leverages on the prior knowledge of the population structure to inform on a relatively parsimonious SNP 
141 set with moderately high prediction. The DT selector, the secondary method, was used to select

142 additional SNPs to append to the Broad barcode and the HFST panel for further enhancement of

143 geographical prediction. A 10-fold cross-validation strategy was used to assess the performance of the

144 selectors with the likelihood-based classification framework.

145 To achieve the secondary objective of the study, identifying single gene regions with moderate-to-high

146 country-level resolution, simulations were run across individual genes using the HFST-0.75 (HFST with

147 FST threshold of 0.75) selector model with the likelihood classifier. The top 20 genes with the highest

148 pooled median Matthew Correlation Coefficient (MCC) scores for each selector model were reported

149 (Figure 1b).

\section{Overview of the web-based data analysis and sharing platform}

152 To establish accessible informatics tools to support users to analyze and interpret their data, a platform

153 was created incorporating data classification tools for determining the most likely country of origin of a

154 sample using genetic data at a given barcode. Existing source code, developed for a microsatellite-based

155 P. vivax data sharing and analysis platform ${ }^{9}$, was modified to create a new web-based platform

156 (vivaxGEN-geo), to collate SNP data generated at the geographic barcode. A likelihood-based classifier

157 approach was chosen for geographic assignment within the vivaxGEN-geo platform owing to the ability

158 to i) incorporate manual SNP sets, ii) evaluate barcodes with missing data, and iii) evaluate heterozygous

159 genotype calls.

162 The custom classifier was developed to handle bi-allelic heterozygote calls for mix-infection cases by

163 treating the samples as diploid samples, as well as missing data by treating as heterozygote calls. The 
164

165

166

167

168

169

170

171 172 samples.

classifier was derived from Bernoulli Naive Bayes with modification to the likelihood equation and elimination of prior probability, since the distribution of our dataset did not reflect the distribution in nature, but rather the implication from sample and extracted DNA quality, as well as the characteristics of the original study such as duration and type of the study. Hence the classifier only depends on the likelihood of the SNP data. The likelihood equation was modified to handle the bi-allelic data as follows:

$$
L(X \mid C k)=\prod_{i}^{n} \quad p_{k i}^{x_{i}} \cdot\left(1-p_{k i}\right)^{\left(2-x_{i}\right)}
$$

where $\boldsymbol{X}$ is the SNP data set of a sample, $C k$ is a group (or a country), $x_{i}$ is the number of alternate alleles at position $i$ and $p_{k i}$ is the frequency of the alternate allele at position $i$ of country $k$ counted as diploid

\section{SNP Selection}

175 To select optimal SNPs for country classification, a combination of the HFST and DT selector methods 176 were employed. The DT selector utilized the Python-based scikit-learn package for the decision tree 177 implementation, which employed an optimized version of the CART (Classification And Regression Tree) 178 algorithm and Gini coefficient. To avoid overfitting, a minimum of 3 samples was required for a leaf 179 node. The Hierarchical FST (HFST) approach worked by traversing across a bifurcating guide tree and 180 selecting SNP with the highest FST between the two populations represented by the two nodes of the

181 branch with the assumption that the SNP with the highest FST might differentiate those two 182 populations. If the highest FST of a certain branch was lower than a given threshold during guide tree 183 traversal, the DT method was employed to obtain additional SNPs to separate the given branch. To 184 avoid overfitting, a maximum tree depth of 2 was set for this particular DT step. The HFST analysis in this 185 study was undertaken using a guide tree constructed using Nei's population distance matrix implemented with a neighbor-joining algorithm. 
187 The classification performance was measured with MCC for each country ${ }^{10}$. In addition, the pooled

188 median, mean, minimum and first-quartile MCC were collected as additional measurements.

189 Three models, HFST-0.90 (HFST and DT with FST threshold of 0.90), HFST-0.95 (HFST and DT with FST

190 threshold of 0.95), and pure DT were trained with the full dataset. For each of the three models, 500

191 repeats were run to allow for different random seeds of the DT analysis, and the top 25 SNP sets with

192 the highest aggregate minimum MCC score as evaluated by the likelihood classifier were obtained from

193 each model. A stratified 100 repeat, 10 -fold cross-validation was run on each of the 25 SNP sets from

194 each model, and the best SNP set from each of model, as indicated by highest aggregate minimum MCC

195 score within a repeat, was selected. To compare the Broad SNP panel to the three new SNP panels

196 identified by the HFST-0.90, HFST-0.95 and pure DT selectors, a 500 repeat, stratified 10 -fold cross-

197 validation was undertaken on each SNP panel.

\section{Missing data evaluation}

To assess the durability of prediction performance of the SNP sets with missing data, a simulation was run by removing genotype data randomly. The Likelihood classifier was trained against the selected SNP sets using all samples. For each country, 25 samples were sampled randomly with replacement and missing genotype calls were added to the SNP sets in $10 \%, 20 \%$ and $30 \%$ proportions. The random samples were then subjected to the trained classifier. This process was run in 100 repeats and MCC-

205 score of the prediction for each country was reported.

\section{Datasets}

208 The analysis included genomic data on P. vivax isolates collected from 26 countries. Published data were 209 included from 19 countries derived from the European Nucleotide Archive ${ }^{11-15}$, and new data from 10 countries (Supplementary Table 1, Supplementary Figure 1). New genomic data were derived from 
211 patients recruited to partner studies in Afghanistan, Bangladesh, Bhutan, Colombia, Ethiopia, Indonesia,

212 Iran, Malaysia, Sudan, and Vietnam. With the exception of Colombia, the patient sampling frameworks

213 have been described previously ${ }^{11,12,14,16-20}$. The samples from Colombia were collected within the

214 framework of cross-sectional epidemiological surveys conducted between 2013 and 2017. Whole

215 genome sequencing, read alignment and variant calling were undertaken within the framework of a $P$.

216 vivax community study in the Malaria Genomic Epidemiology Network (MalariaGEN) ${ }^{21}$. Data was

217 derived from an open dataset of Plasmodium vivax genome variation comprising 2,671,112 discovered

218 variants across 1,366 isolates (MalariaGEN manuscript in preparation). The data were initially filtered to

219 derive a set of 670,962 high-quality bi-allelic SNPs with VQSLOD score $>0$, and minimum read depth and

220 minimum minor allele count of 2. Individual genotype calls were defined as heterozygotes based on an

221 arbitrary threshold of a minor allele ratio $>0.1$ and a minimum of 2 reads for each allele; all other

222 genotype calls were defined as homozygous for the major allele. A pair of isolates with distance less

223 than $0.0005(0.05 \%)$ were considered non-independent. Amongst non-independent sample pairs, the

224 isolate with the higher percentage of genotype failures was removed from the dataset; this removal

225 process was iterated until all non-independent isolates had been removed from the dataset. The

226 samples and SNPs were then subjected to further filtering to eliminate missing data using information

227 derived from a simulation which calculated the total number of SNPs with no missing data and the

228 number of consecutive informative SNPs as defined by SNPs with minimum minor allele count $($ MAC) $>2$.

229 The remaining dataset was defined as Dataset 1.

230 The isolates in Dataset 1 were initially assigned to national groups based on the country in which the

231 patient presented at the clinic with the infection. The national-level groupings were evaluated further

232 using country-level assignments derived from the genome-wide data classification with the likelihood

233 classifier. Infections presenting with country classifications differing from the country of presentation

$234 \quad$ were considered suspected imported infections and removed to produce Dataset 2. 
Of the 42 Broad barcode SNPs, 37 SNPs were present in the 670K dataset (bi-allelic high-quality SNPs) and exhibited successful amplicon-based sequencing assays (personal communication, Wellcome Sanger

237 Institute Core Sequencing Facility); these 37 SNPs were not present in dataset 1 or 2 . A new dataset

238 (Dataset 3) was prepared for evaluation of the Broad barcode comprising of samples with complete data

239 across the 37 Broad barcode SNPs and partial data across SNPs selected from the HFST and DT

240 algorithms.

\section{Software and Web Service Availability}

243 All custom, in-house scripts used for data filtering, simulation, analyses and visualization are available

244 from http://github.com/trmznt/vivaxgen-geo. The VivaxGEN-geo web service provides a user-friendly

245 online tool for country classification with all SNP sets, and is accessible at https://geo.vivaxgen.org/. The

246 likelihood classifier provided on the online platform has been trained with 809 samples (dataset 4),

247 consisting of all samples with complete data at all SNP sets. The classifier tool reports the three highest

248 likelihoods for country of origin and their associated probabilities.

\section{Ethics}

251 All samples were collected with written informed consent from patients or their legal guardians. Ethical

252 approvals for the published samples are detailed in the original papers ${ }^{11-15}$. Approvals for the newly 253 represented studies are outlined in Supplementary Document 1.

\section{Results}

\section{Geographic clustering patterns using the genome-wide dataset}

257 The primary dataset (Dataset 1) was derived using the missing data simulation, to minimise genotype

258 failures (Supplementary Figure 3), it comprised 854 high-quality samples and 294,628 high-quality 
informative SNPs, with no missing data. The median percentage of heterozygous calls in each country ranged from $0.02 \%$ to $0.08 \%$. Details on the geographic locations of the samples in dataset 1 are clustering of most countries (Supplementary Figure 4). Exceptions included the isolates from countries, the majority of isolates in these countries could be differentiated by national boundaries.

267 Visual inspection of the neighbour-joining tree revealed potential imported cases. Using country-level assignments derived from the genome-wide data classification with the likelihood classifier and manual confirmation of the neighbor-joining tree, 21 isolates presented country classifications differing from the country of presentation (Supplementary Table 1). After exclusion of the imported cases, and countries

272 (Supplementary Table 1).

Performance of the Broad barcode, HFST and DT SNP selection

275 When the HFST selector was applied with an FST threshold of 0.90 (HFST-0.90), a set of 28 SNPs (listed in

276 Supplementary Table 2) were identified. This dataset exhibited median MCC scores exceeding 0.9 in all

277 countries with the exception of Vietnam (0.75) and Cambodia (0.80). On increasing the FST threshold to 2780.95 (HFST-0.95), the HFST model identified 51 SNPs (listed in Supplementary Table 3), which displayed

279 MCC scores exceeding 0.95 in all countries except for Vietnam (0.85) and Cambodia (0.87). Using the DT 280 selector alone, 50 SNPs (listed in Supplementary Table 4) displayed comparable performance to the 51-

281 SNP panel, with a slightly lower aggregate minimum MCC score.

282 The results of cross-validation of the classification performance of the five SNP panels (37-SNP Broad barcode, 28-SNP, 28-SNP plus Broad barcode (65-SNP), 50-SNP and 51-SNP panels) are illustrated in 
284 Figure 2, and the MCC and F scores reflecting the consensus results of the cross-validation are

285 summarized in Table 1. The performance, ranked from lowest to highest, was: 37-SNP Broad barcode

286 (median MCC = 0.82), 28-SNP (MCC = 0.99), 50-SNP (MCC = 1.00), 65-SNP (MCC = 1.00), and 51-SNP

$287 \quad(\mathrm{MCC}=1.00)$.

288

Missing data simulations

290

291

292

293

294

295

296

297

298

299

300

301

302

303

304

305

306

307

In the missing data simulations, genotyping failures had the greatest impact on the classification of samples from Cambodia and Vietnam (Figure 3). With 10\% missing data, the median MCCs of the 28-SNP panel exceeded 0.9 in all countries, with exception of Vietnam $(\mathrm{MCC}=0.80)$ and Cambodia $(\mathrm{MCC}=$ 0.77). With this level of missing data, the addition of the 37 Broad SNPs (65-SNP panel) increased the median MCC to 0.83 in Vietnam and 0.82 in Cambodia. When missing data increased to $30 \%$, the 65 -SNP panel achieved median MCCs above 0.9 in most countries, with exception of Vietnam (MCC $=0.79)$ and Cambodia ( $M C C=0.75)$. The 50- and 51-SNP panels both achieved MCC scores exceeding 0.95 for all countries except Cambodia (0.80-0.82) and Vietnam (0.83-0.85) with 10-30\% missing data.

\section{Evaluation of single gene regions to predict country classification}

The suitability of single genes to predict country classifications were assessed by simulations of individual genes using HFST-0.75 selector model with the likelihood classifier framework. The top 20 genes with the highest pooled median MCC scores for the HFST-0.75 are presented in Supplementary Table 5. The highest prediction capacity, with median MCC score of 0.68, was PVP01_0302600, a gene coding a $11.5 \mathrm{~Kb}$ conserved protein with unknown function. The gene list also included three members of the cysteine repeat modular protein family (CRMP): CRMP1 (median MCC $=0.63), \mathrm{CRMP3}(\mathrm{MCC}=$ $0.57)$ and $\mathrm{CRMP} 4(\mathrm{MCC}=0.56)$. 


\section{Discussion}

309 The primary objective of the study was to develop molecular tools amenable to population-based

310 surveillance frameworks to identify and map imported $P$. vivax infections. Using machine-learning

311 methods, 3 new SNP panels were identified with high country classification performance, able to

312 distinguish imported $P$. vivax infections across a range of endemic scenarios. The most parsimonious

313 panel, the 28-SNP barcode, exhibited high country classification, and can be appended to the $37 \mathrm{bi}$ -

314 allelic, assayable Broad barcode SNPs for marginal improvement in predictive capacity in samples with

315 moderate levels of missing data. The combined 65-SNP barcode generated robust country classification

316 in most endemic areas, even when the proportion of missing data rose to $30 \%$. However, the validity of

317 the 65-SNP barcode was lower in Cambodia and Vietnam, a likely reflection of the porous border

318 between these two countries. Although the 50- and 51-SNP panels achieved better resolution in these

319 areas, characterization of parasite transmission across borders with high levels of gene flow may be

320 addressed better by the addition of markers suited to an analysis of identity-by-descent ${ }^{22}$. The

321 application and wider validation of the 65-SNP barcode is underway, with amplicon-based sequencing

322 assays already established for the 37 Broad barcode SNPs, and under development for the 28 new

323 markers.

324 The analysis and interpretation of "real-world" genotyping data raises significant challenges from low-

325 quality samples such as those collected on dried blood spots. In anticipation of these needs we

326 established a likelihood-based framework with the capacity to deal with polyclonal infections as well as

327 missing data. This framework has been integrated into the vivaxGEN-geo online platform, so that users

328 can analyze and interpret their data without needing complex bioinformatics skills and avoiding the

329 subjective visual inspection of neighbour-joining trees or principal component plots. Whilst the

330 informatics tools implemented in vivaxGEN-geo are tailored to $P$. vivax, we anticipate that a similar

331 approach can be adapted to other species. To facilitate wider application the source code will be made

332 publicly available. 
333 The variants in the 28-SNP panel are located in genes representing a range of functions, some of which

334 may be unstable over time. Although our dataset represents one of the most geographically diverse

335 panels of $P$. vivax isolates currently available, with representation of all of the major vivax-endemic

336 regions, the predictive capacity of the derived tools are likely to be constrained by the geographic

337 representation of the reference panel. In particular, representation from central and south America and

338 the Indian subcontinent were limited in our data set. Despite this limitation the dataset used comprises

339 good representation of isolates from areas of public health relevance, including the epicenter of

340 chloroquine-resistant $P$. vivax in Papua Indonesia ${ }^{23,24}$. The likelihood-based framework is able to re-

341 evaluate the predictive potential of current marker sets as new genomic data become available, so that

342 the selected SNP panels can be refined further in an iterative process. Furthermore, as the reference

343 panel expands with increasing data generated at the barcode SNPs, the accuracy of the likelihood-based

344 classifications will improve.

345 In addition to the independent selection of SNPs, a number of informative genes were identified, each

346 of which had moderately high geographic resolution power. Genotyping of these genes or gene regions

347 are amenable to standard capillary sequencing, offering an alternative approach, albeit with slightly

348 lower resolution, to define a parasite's geographic origin in settings where high-throughput genotyping

349 facilities are unavailable. The genes with the greatest geographic resolution, included members of the

350 cysteine repeat modulator protein (CRMP) family (CRMP1, CRMP3 and CRMP4) implicated in essential

351 roles in parasite transmission from the mosquito to the human host ${ }^{25}$. It is plausible that the CRMP

352 genes have maintained high geographic differentiation to ensure parasite adaptation to the local vector

353 species. Although adaptations of these genes are likely to temporally stable, the resolution of these loci

354 may be constrained by the distributions of host Anopheles vector species.

355 In 2017, up to $100 \%$ of all confirmed malaria cases in 17 malaria-endemic countries in the Asia-Pacific

356 region, the Middle East and the Americas, where $P$. vivax infections predominate, were reported as

357 being infections ${ }^{1}$. Malaria control programs in these countries can utilize the information derived from 
the molecular tools provided from our analysis to assess the efficacy of ongoing interventions in

reducing local transmission, and to determine the major routes of infection importation. The tools have

potential to reduce ambiguity for certificating malaria elimination by the World Health Organization,

where one of the key requirements is the demonstration that all malaria cases detected in-country over

at least three consecutive years were imported. For this purpose, countries approaching elimination will

need to maintain archival samples for future molecular comparisons against putatively imported cases.

The molecular $P$. vivax geographic classification tools presented are designed to empower users in

malaria-endemic countries to analyze and interpret locally produced genotyping data with comparison

to globally available datasets. Amplicon-based sequencing of the full 65-SNP barcode is being developed

367 and will be combined with other surveillance markers at central laboratories in endemic partner

\section{References}

374 1. WHO. World Malaria Report 2018. World Health Organization; Geneva 2018. (2018).

375 2. White, N.J. \& Imwong, M. Relapse. Adv Parasitol 80, 113-50 (2012).

3763 3. Tripura, R. et al. Persistent Plasmodium falciparum and Plasmodium vivax infections in a

377 western Cambodian population: implications for prevention, treatment and elimination

$378 \quad$ strategies. Malar J 15, 181 (2016).

379 4. Sattabongkot, J., Tsuboi, T., Zollner, G.E., Sirichaisinthop, J. \& Cui, L. Plasmodium vivax transmission: chances for control? Trends Parasitol 20, 192-8 (2004).

5. Iwagami, M. et al. Geographical origin of Plasmodium vivax in the Republic of Korea: haplotype network analysis based on the parasite's mitochondrial genome. Malar J 9, 184 (2010).

6. Rodrigues, P.T. et al. Using mitochondrial genome sequences to track the origin of imported Plasmodium vivax infections diagnosed in the United States. Am J Trop Med Hyg 90, 1102-8 (2014).

7. Diez Benavente, E. et al. Genomic variation in Plasmodium vivax malaria reveals regions under selective pressure. PLoS One 12, e0177134 (2017). 
400

401

402

403

404

405

406

407

408

409

410

411

412

413

414

415

416

417

418

419

420

421

422

423

424

425

426

427

428

429

430

431

8. Baniecki, M.L. et al. Development of a single nucleotide polymorphism barcode to genotype Plasmodium vivax infections. PLoS Negl Trop Dis 9, e0003539 (2015).

9. Trimarsanto, H. et al. VivaxGEN: An open access platform for comparative analysis of short tandem repeat genotyping data in Plasmodium vivax Populations. PLoS Neg/ Trop Dis 11, e0005465 (2017).

10. Jurman, G., Riccadonna, S. \& Furlanello, C. A comparison of MCC and CEN error measures in multi-class prediction. PLoS One 7, e41882 (2012).

11. Auburn, S. et al. Genomic analysis of a pre-elimination Malaysian Plasmodium vivax population reveals selective pressures and changing transmission dynamics. Nat Commun 9, 2585 (2018).

12. Auburn, S. et al. Genomic analysis of Plasmodium vivax in southern Ethiopia reveals selective pressures in multiple parasite mechanisms. J Infect Dis (2019).

13. Hupalo, D.N. et al. Population genomics studies identify signatures of global dispersal and drug resistance in Plasmodium vivax. Nat Genet 48, 953-8 (2016).

14. Pearson, R.D. et al. Genomic analysis of local variation and recent evolution in Plasmodium vivax. Nat Genet 48, 959-64 (2016).

15. Parobek, C.M. et al. Selective sweep suggests transcriptional regulation may underlie Plasmodium vivax resilience to malaria control measures in Cambodia. Proc Natl Acad Sci U S A 113, E8096-E8105 (2016).

16. Wangchuk, S. et al. Where chloroquine still works: the genetic make-up and susceptibility of Plasmodium vivax to chloroquine plus primaquine in Bhutan. Malar J 15, 277 (2016).

17. Ley, B. et al. G6PD Deficiency and Antimalarial Efficacy for Uncomplicated Malaria in Bangladesh: A Prospective Observational Study. PLoS One 11, e0154015 (2016).

18. Hamedi, Y. et al. Molecular Epidemiology of P. vivax in Iran: High Diversity and Complex SubStructure Using Neutral Markers, but No Evidence of Y976F Mutation at pvmdr1. PLoS One 11, e0166124 (2016).

19. Getachew, S. et al. Variation in Complexity of Infection and Transmission Stability between Neighbouring Populations of Plasmodium vivax in Southern Ethiopia. PLoS One 10, e0140780 (2015).

20. Taylor, W.R.J. et al. Short-course primaquine for the radical cure of Plasmodium vivax malaria: a multicentre, randomised, placebo-controlled non-inferiority trial. Lancet (2019).

21. Malaria Genomic Epidemiology, N. A global network for investigating the genomic epidemiology of malaria. Nature 456, 732-7 (2008).

22. Taylor, A.R., Jacob, P.E., Neafsey, D.E. \& Buckee, C.O. Estimating Relatedness Between Malaria Parasites. Genetics 212, 1337-1351 (2019).

23. Ratcliff, A. et al. Therapeutic response of multidrug-resistant Plasmodium falciparum and $P$. vivax to chloroquine and sulfadoxine-pyrimethamine in southern Papua, Indonesia. Trans $R$ Soc Trop Med Hyg 101, 351-9 (2007).

24. Price, R.N. et al. Global extent of chloroquine-resistant Plasmodium vivax: a systematic review and meta-analysis. Lancet Infect Dis 14, 982-91 (2014).

25. Douradinha, B. et al. Plasmodium Cysteine Repeat Modular Proteins 3 and 4 are essential for malaria parasite transmission from the mosquito to the host. Malar J10, 71 (2011).

26. Battle, K.E. et al. Mapping the global endemicity and clinical burden of Plasmodium vivax, 200017: a spatial and temporal modelling study. Lancet 394, 332-343 (2019).

\section{Acknowledgements}


433 We would like to thank the patients who contributed their samples to the study, local health facilities,

434 and the health workers and field teams who assisted with the sample collections. We also thank the

435 staff of the Wellcome Sanger Institute Sample Logistics, Sequencing, and Informatics facilities for their

436 contributions.

\section{Financial Support}

439 Financial support for the study was provided by the Wellcome Trust (Senior Fellowship in Clinical

440 Science awarded to R.N.P., 200909), the Australian Department of Foreign Affairs and Trade (TDCRRI

441 72904), the Australian National Health and Medical Research Council (NHMRC) ('Improving Health

442 Outcomes in the Tropical North: A multidisciplinary collaboration ‘HOT North' Career Development

443 Fellowship awarded to S.A.), and the Bill and Melinda Gates Foundation (OPP1164105). D.F.E received

444 financial support from Colciencias -Colombia, call 656-2014 "Es Tiempo de Volver" award FP44842-503-

445 2014. The patient sampling and metadata collection was funded by the Asia-Pacific Malaria Elimination

446 Network (108-07), the Malaysian Ministry of Health (BP00500420), and the NHMRC (1037304 and

447 1045156; Fellowships to N.M.A. [1042072 and 1135820], B.E.B. [1088738] and M.J.G. [1074795]). M.J.G

448 was also supported by a 'Hot North' Earth Career Fellowship (1131932). M.U.F is supported by a senior

449 researcher scholarship from the Conselho Nacional de Desenvolvimento Científico e Tecnológico (CNPq),

450 Brazil. The whole genome sequencing component of the study was supported by grants from the

451 Medical Research Council and UK Department for International Development (M006212) and the

452 Wellcome Trust $(206194,204911)$ awarded to D.P.K. This work was supported by the Australian

453 Centre for Research Excellence on Malaria Elimination (ACREME), funded by the NHMRC (APP

454 1134989). 


\section{$456 \quad$ Figures}

457 Figure 1. Overview of the marker selection approaches

458 Flow diagrams illustrating the datasets, selector models and classification approaches used to identify

459 and evaluate independent SNP panels (A) and single gene regions (B). Decision Tree (DT), HFST-0.90

460 (HFST and DT with FST threshold of 0.90), HFST-0.95 (HFST and DT with FST threshold of 0.95) and HFST-

4610.75 (HFST and DT with FST threshold of 0.75) represent the SNP selector models. The DT, HFST-0.9 and

462 HFST-0.95 SNP selector models were run in 500 repeats for SNP selection (A), and the HFST-0.75 model

463 was run in 5 repeats for gene selection (B). For SNP selection (A), the top-25 SNP sets were selected

464 from each model for a further 100 repeats of stratified cross-validation from which one SNP set was

465 selected from each of the DT, HFST-0.9 and HFST-0.95 SNP selector models.

Figure 2. Comparison between the 37-SNP Broad barcode, new marker panels and combined SNP sets

The Broad-37 SNP set reflects 37 of the 42 Broad SNPs represented amongst the 294K high-quality SNPs.

469 The SNP-28 SNP set reflects 28 high-performance SNPs derived from the HFST selector with FST

470 threshold of 0.9. The SNP-28+Broad SNP set reflects the combined Broad-37 and SNP-28 SNP sets for a

471 total of 65 SNPs. The SNP-50 set reflects the 50 SNPs selected by the Decision Tree selector. The SNP-51

472 set reflects 51 high-performance SNPs from the HFST selector with threshold FST of 0.95 . The boxplots

473 present the MCC scores from 500 repeats with stratified 10 -fold cross validation for each SNP set.

474 Country labels are provided on the y-axis; MEDIAN, MEAN, Q1 (1st percentile) and MIN reflect the

475 respective summary statistics for the pooled MCC scores across all countries. 
478 Result of 200 repeats, 25 samples per country simulation of missing data (genotype fails) of $10 \%, 20 \%$

479 and $30 \%$ against the 37-SNP Broad barcode, 28-SNP set, 65-SNP set (28-SNP + Broad panel), 51-SNP and

480 50-SNP set. The 65-SNP set demonstrated marginally better performance relative to the 28-SNP set with

481 missing data. However, both the 50-SNP and 51-SNP panels outperformed the 65-SNP panel with

482 missing data.

Supplementary Figure 1.P. vivax prevalence map pinpointing the countries included in the study

P. vivax prevalence map from the Malaria Atlas Project (Plasmodium vivax parasite rate in all ages

486 globally (1-99) from $(2000-2017)^{26}$, with counties included in dataset 2 demarked by stars.

\section{Supplementary Figure 2. Overview of the datasets}

\section{Supplementary Figure 3. Output from the data quality simulation}

491 The upper panel shows the number of complete SNPs (green), complete informative SNPs with minor

492 allele count $(\mathrm{MAC})=1$ (orange) and complete informative SNPs with MAC $=2$ (red) against the number 493 of samples. The lower panel shows the number of differences in SNPs between consecutive number of 494 samples, with informative SNPs with MAC = 1 (blue) and informative SNPs with MAC = 2 (orange). The 495 maximum of both $M A C=1$ and $M A C=2$ were 958 samples.

498 The tree was constructed using genotyping data from 854 samples at 294K SNPs. 


\section{Tables}

Table 1. Summary of MCC and F-scores from the consensus results of 500 repeats of the stratified 10-fold cross-validation of the SNP panels

\begin{tabular}{|c|c|c|c|c|c|c|c|c|c|c|}
\hline \multirow[t]{2}{*}{ Population } & \multicolumn{2}{|c|}{ 37-SNP (Broad) } & \multicolumn{2}{|c|}{ 28-SNP } & \multicolumn{2}{|c|}{ 65-SNP } & \multicolumn{2}{|c|}{ 50-SNP } & \multicolumn{2}{|c|}{ 51-SNP } \\
\hline & MCC & $\mathbf{F}$ & MCC & $\mathbf{F}$ & MCC & $\mathbf{F}$ & MCC & $\mathbf{F}$ & MCC & $\mathbf{F}$ \\
\hline Afghanistan & 0.852 & 0.857 & 0.987 & 0.988 & 1 & 1 & 0.974 & 0.975 & 0.987 & 0.988 \\
\hline Bangladesh & 0.796 & 0.778 & 0.881 & 0.875 & 1 & 1 & 1 & 1 & 1 & 1 \\
\hline Bhutan & 0.665 & 0.615 & 1 & 1 & 1 & 1 & 0.894 & 0.889 & 1 & 1 \\
\hline Brazil & 0.815 & 0.8 & 1 & 1 & 1 & 1 & 1 & 1 & 1 & 1 \\
\hline Cambodia & 0.456 & 0.518 & 0.78 & 0.809 & 0.813 & 0.837 & 0.893 & 0.907 & 0.898 & 0.911 \\
\hline China & 0.912 & 0.909 & 1 & 1 & 1 & 1 & 1 & 1 & 1 & 1 \\
\hline Colombia & 0.895 & 0.903 & 1 & 1 & 1 & 1 & 1 & 1 & 1 & 1 \\
\hline Ethiopia & 0.929 & 0.931 & 1 & 1 & 1 & 1 & 1 & 1 & 1 & 1 \\
\hline India & 0.714 & 0.706 & 1 & 1 & 1 & 1 & 0.935 & 0.933 & 0.925 & 0.923 \\
\hline Indonesia & 0.819 & 0.857 & 0.971 & 0.978 & 0.984 & 0.988 & 0.987 & 0.99 & 0.99 & 0.993 \\
\hline Iran & 0.865 & 0.857 & 0.925 & 0.923 & 1 & 1 & 1 & 1 & 1 & 1 \\
\hline Madagascar & 0.894 & 0.889 & 1 & 1 & 1 & 1 & 1 & 1 & 1 & 1 \\
\hline Malaysia & 0.614 & 0.627 & 0.923 & 0.927 & 0.949 & 0.95 & 0.962 & 0.963 & 0.962 & 0.963 \\
\hline Mexico & 0.92 & 0.919 & 1 & 1 & 0.92 & 0.919 & 1 & 1 & 1 & 1 \\
\hline Myanmar & 0.529 & 0.48 & 0.835 & 0.824 & 0.881 & 0.875 & 0.935 & 0.933 & 1 & 1 \\
\hline Papua New Guinea & 0.616 & 0.6 & 0.888 & 0.889 & 0.934 & 0.933 & 0.976 & 0.977 & 0.975 & 0.976 \\
\hline Peru & 0.839 & 0.846 & 1 & 1 & 0.961 & 0.962 & 1 & 1 & 1 & 1 \\
\hline Sudan & 1 & 1 & 1 & 1 & 1 & 1 & 1 & 1 & 1 & 1 \\
\hline Thailand & 0.681 & 0.725 & 0.971 & 0.975 & 0.985 & 0.988 & 0.99 & 0.992 & 0.985 & 0.987 \\
\hline Vietnam & 0.389 & 0.446 & 0.714 & 0.74 & 0.733 & 0.757 & 0.861 & 0.872 & 0.866 & 0.876 \\
\hline Mean & 0.76 & 0.763 & 0.944 & 0.946 & 0.958 & 0.96 & 0.97 & 0.972 & 0.979 & 0.981 \\
\hline Median & 0.817 & 0.823 & 0.994 & 0.994 & 1 & 1 & 0.995 & 0.996 & 1 & 1 \\
\hline Q1 & 0.653 & 0.624 & 0.914 & 0.915 & 0.945 & 0.946 & 0.955 & 0.956 & 0.983 & 0.984 \\
\hline Min & 0.389 & 0.446 & 0.714 & 0.74 & 0.733 & 0.757 & 0.861 & 0.872 & 0.866 & 0.876 \\
\hline
\end{tabular}


A

\section{SNP Selection}

B

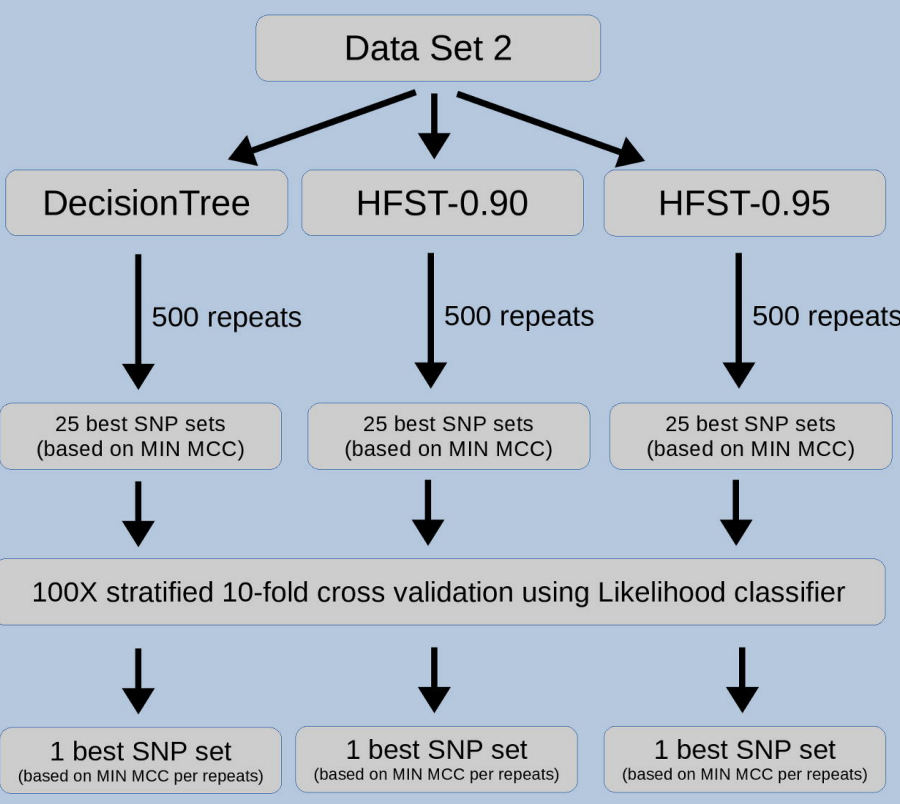

\section{Gene Selection}

\section{Data Set 2}

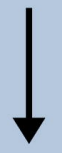

Select gene region

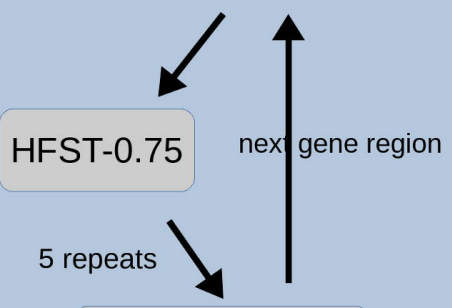

Collect 1 best SNP set (based on MIN MCC) 


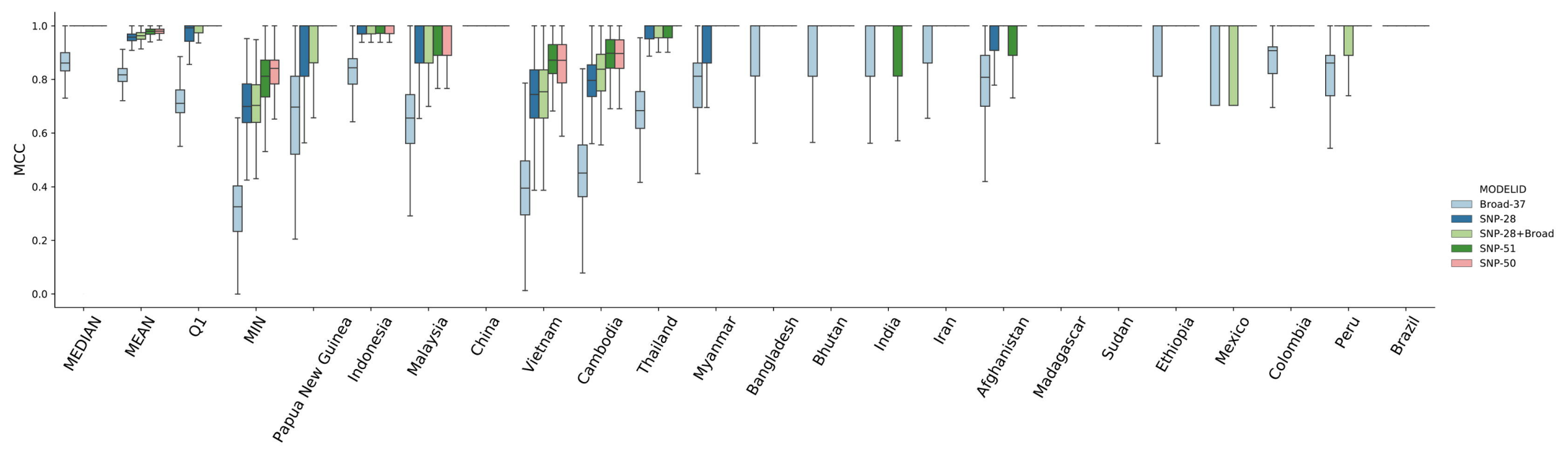



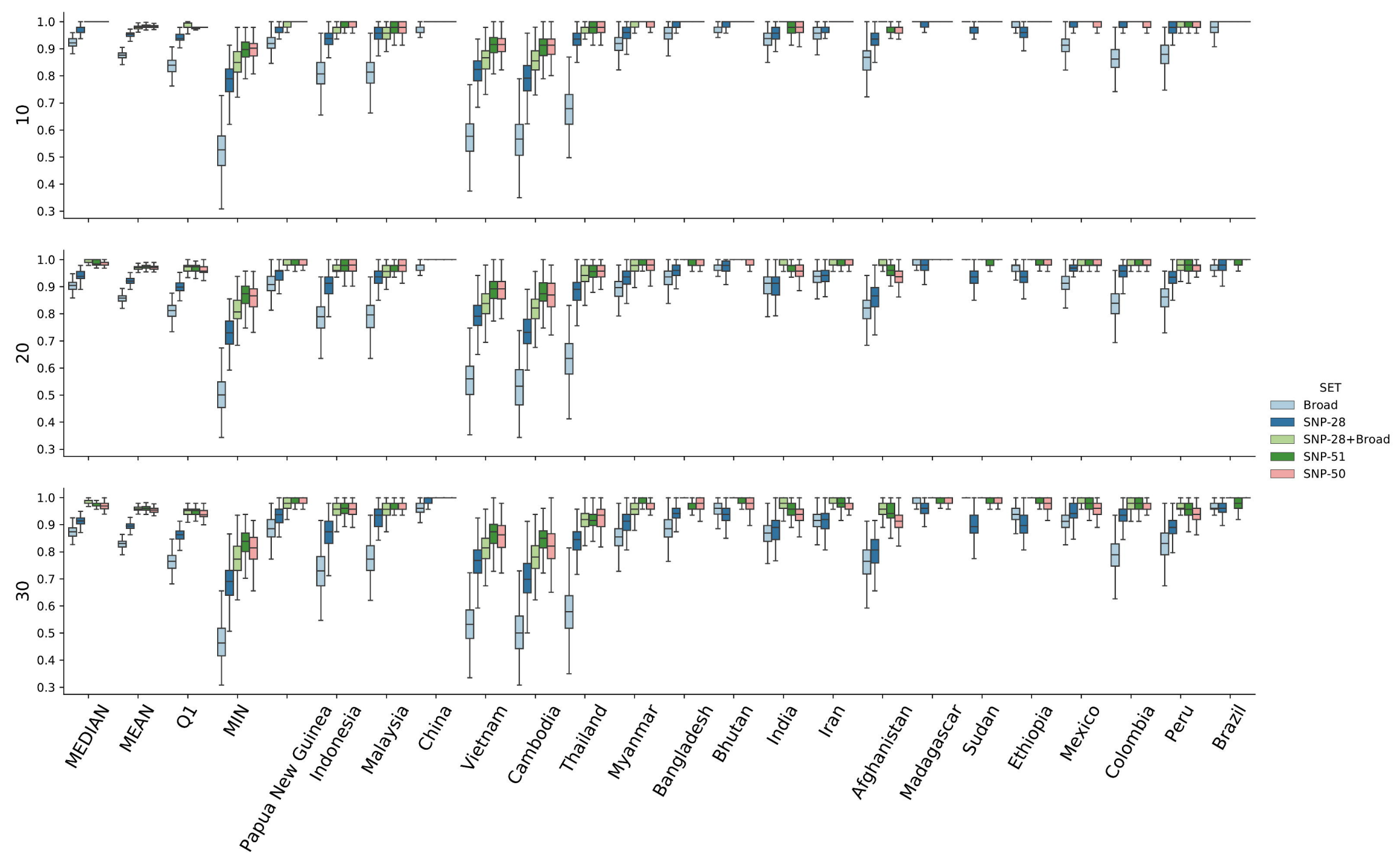

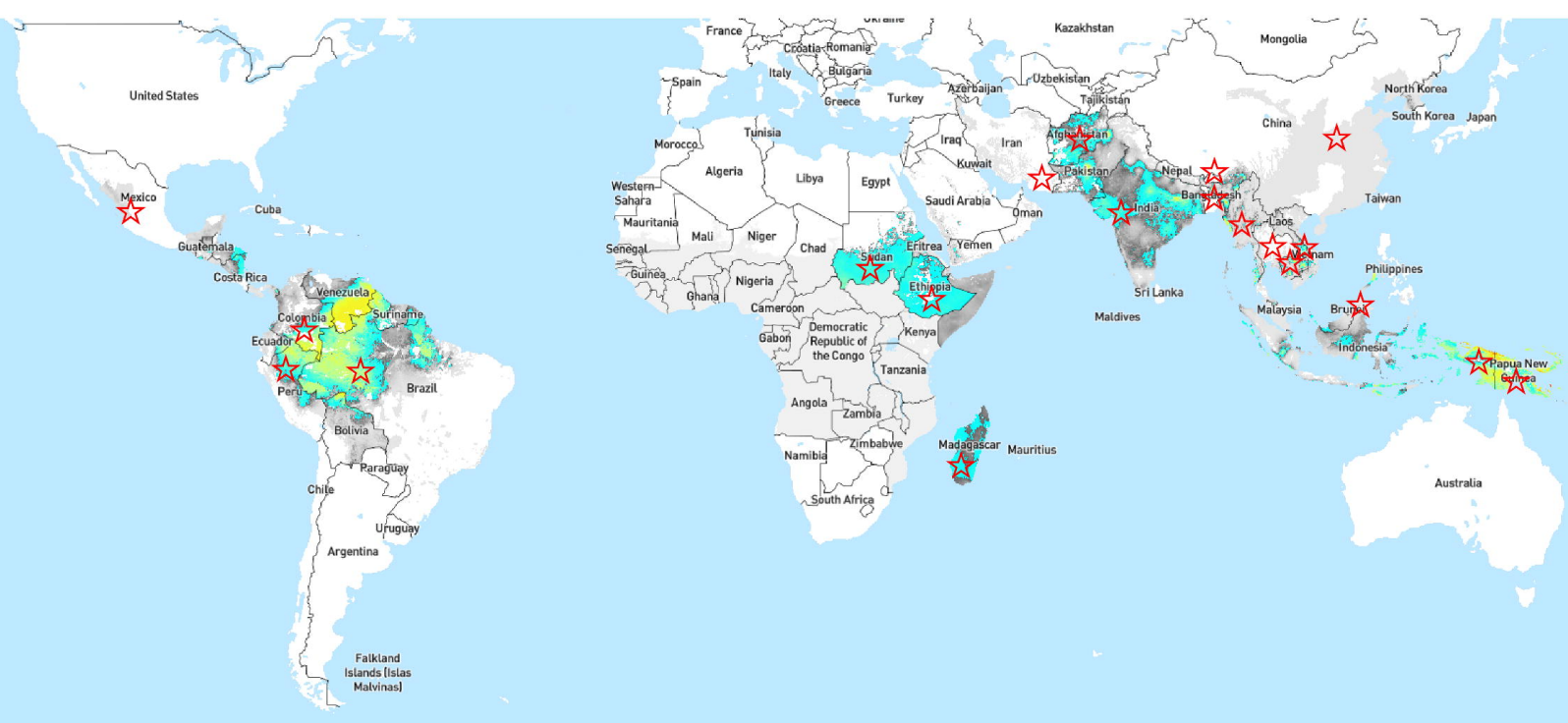


\section{Sample Processing}

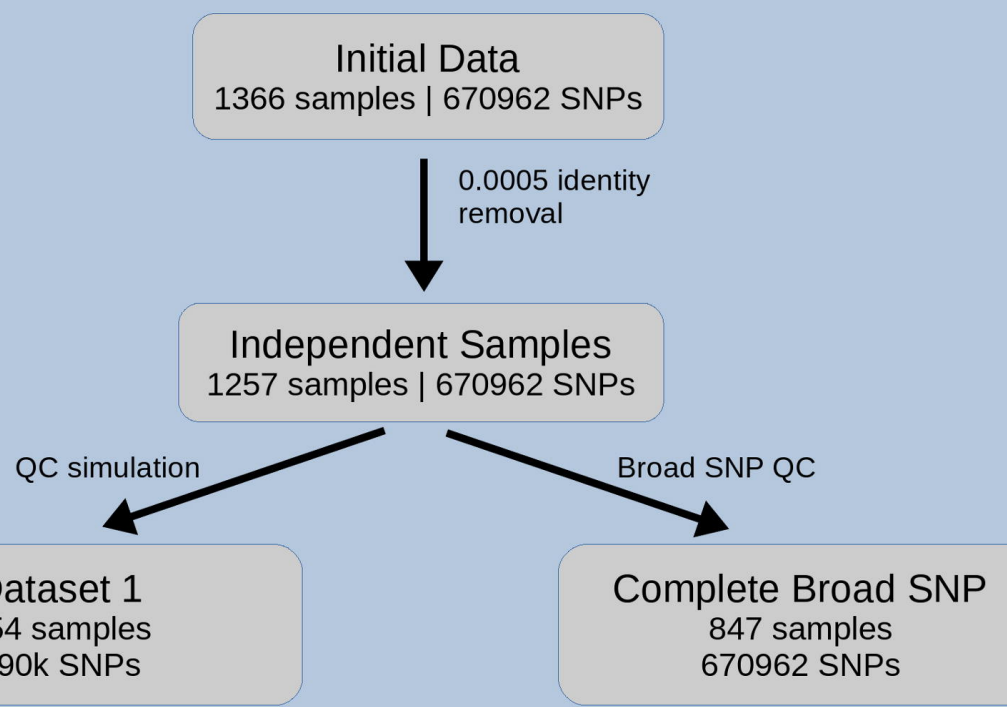

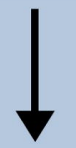

Removal of ambiguous samples and single sample-countries
Dataset 2 831 samples 290k SNPs

Dataset 2
831 samples
290k SNPs

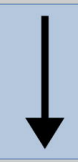

Dataset 3 825 samples

Broad + DT + HFST SNPS

All SNP QC

Dataset 4 809 samples

Broad + DT + HFST SNPS 

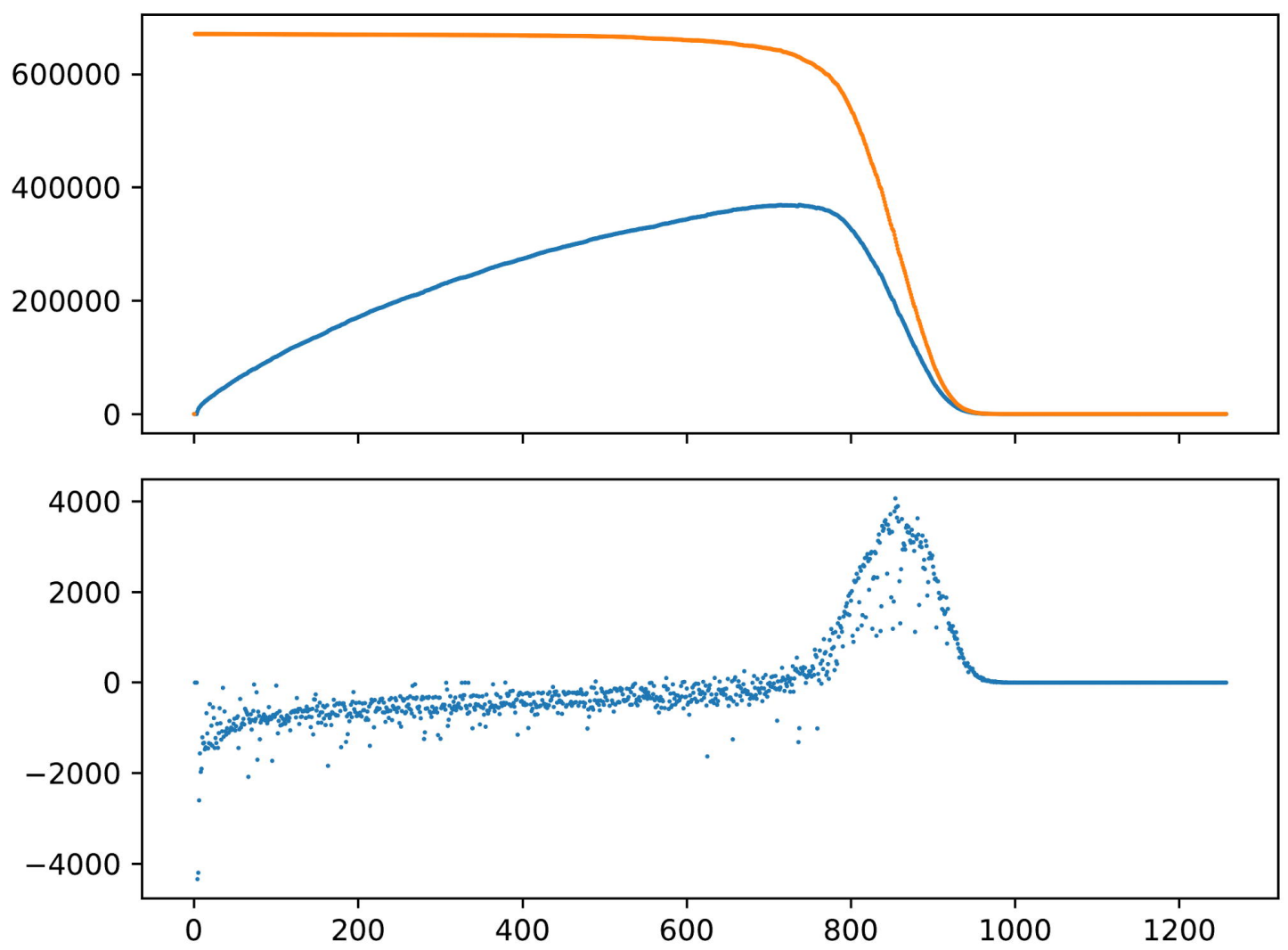


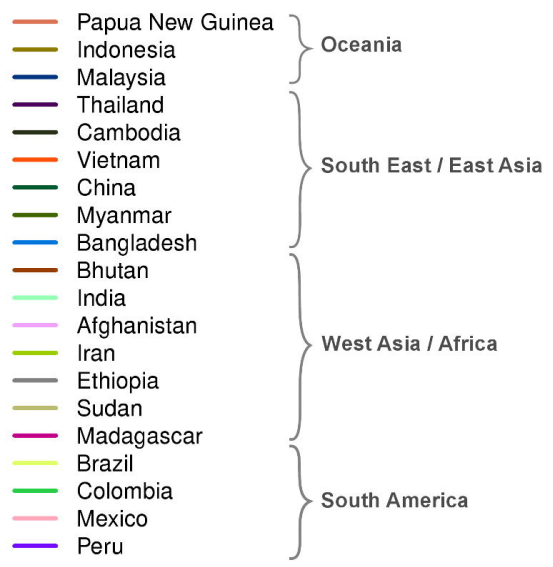

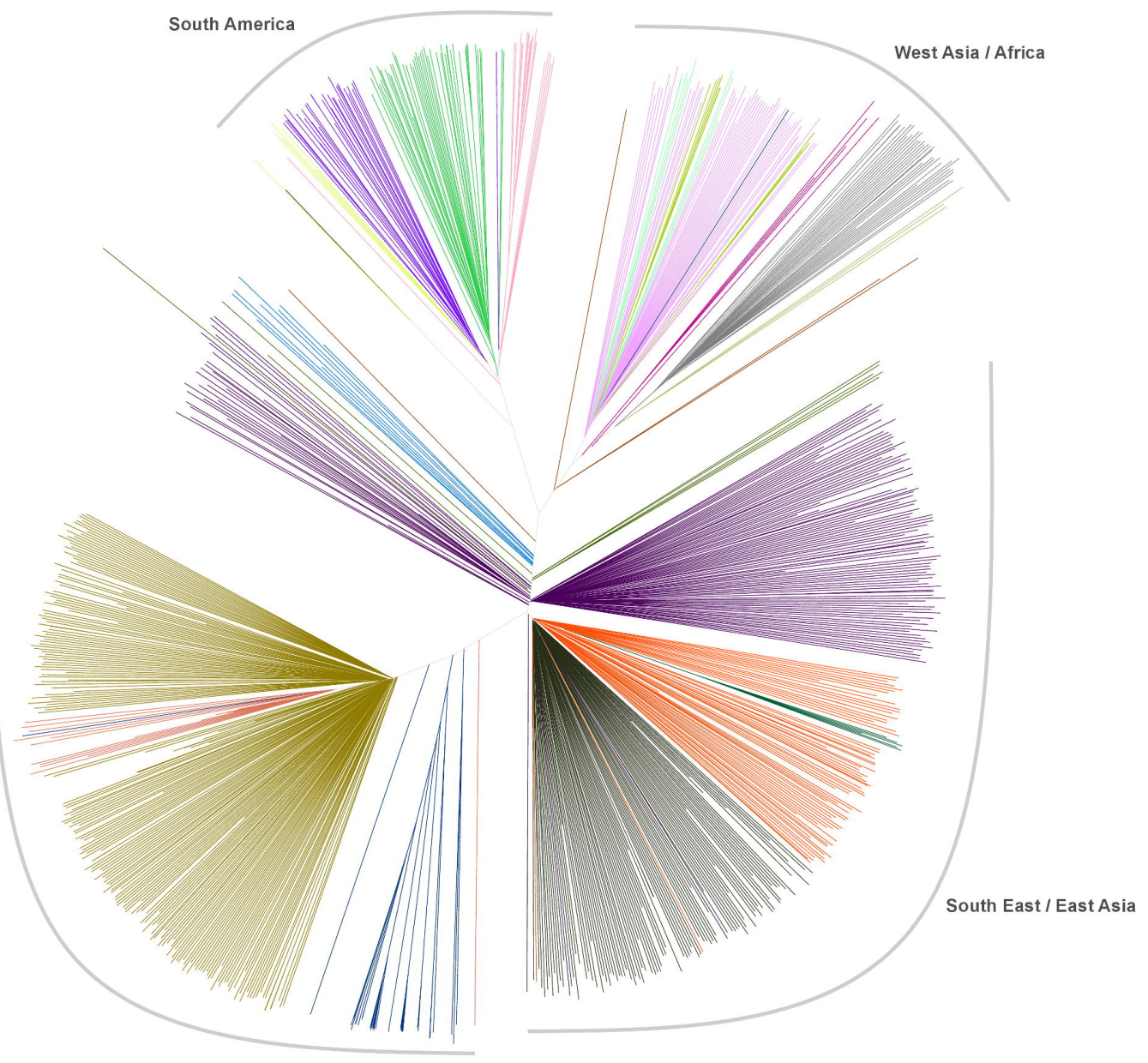

